\title{
Construcción sociodemográfica en Córdoba (Argentina): cambios de las estructuras sociales en el territorio y su impacto en la demanda de hábitat urbano
}

\author{
Sociodemographic construction in Córdoba (Argentina): \\ changes of social structures in the territory and their impact \\ on the demand for urban habitat
}

Sara María Boccolini

\section{Resumen}

Se reconstruyó la situación de Córdoba (Argentina) en cuanto a tendencias sociodemográficas en el territorio en un escenario futuro tendencial a corto plazo, para definir las diferentes demandas de hábitat urbano actuales. Se combinaron variables demográfico/territoriales de información censal oficial, junto con variables de hábitos de vida y de consumo relevados de informes específicos realizados por consultoras públicas y privadas. Esto se complementó con un análisis sociológico basado en la revisión de bibliografía específica. Se detectaron procesos simultáneos de macrocefalia y contraurbanización, así como cambios en estructuras familiares y estilos de vida característicos de la dialéctica entre transición una condición demográfica avanzada y los procesos propios del neoliberalismo posmoderno actuales.

Palabras clave: Córdoba; transición demográfica avanzada; hábitat urbano; macrocefalia; contraurbanización.

\begin{abstract}
The situation in Córdoba (Argentina) is revisited in terms of sociodemographic trends in the territory in a future short-term scenario. Demographic/territorial variables from official census data were combined, along with lifestyle and consumption variables surveyed by public and private consultants. This was complemented by a sociological analysis based on a review of the specific literature. Simultaneous macrocephaly and counterurbanization processes were detected, as well as changes in family structures and lifestyles, characteristic of the dialectic between the condition of an advanced demographic transition and processes of the current postmodern neoliberalism.
\end{abstract}

Keywords: Córdoba; advanced demographic transition; urban habitat; macrocephaly; counterurbanization. 


\section{Introducción}

Este trabajo reconstruye la situación en la ciudad de Córdoba, Argentina, en cuanto a sus tendencias sociodemográficas en un escenario futuro tendencial a corto plazo (10 a 15 años). La estructura sociodemográfica de Argentina y su relación con el territorio funciona como marco referencial de los procesos demográficos de Córdoba para definir las diferentes demandas de hábitat urbano actuales, de acuerdo a los grupos socio-económicos detectados. La ciudad refleja las grandes tendencias de desarrollo demográfico-territorial nacionales, tales como la concentración "macrocefálica", el fuerte aporte migratorio, la estructura etaria y los profundos cambios económicos y culturales que se observan en la composición estructural de los hogares, propios de una etapa de transición demográfica avanzada.

Pero más allá de las correspondencias, este análisis define las particularidades de Córdoba, como son el crecimiento de la población entre 18 y 35 años debido a migraciones nacionales y regionales y renovación permanente, la contraurbanización en el Área Metropolitana de Córdoba (AMCBA), la atomización de la estructura de los hogares y el aumento de grupos familiares de los que una persona es parte a lo largo de su vida, así como la creciente movilidad laboral.

La reconstrucción se realizó combinando variables demográficas y de identificación de estilos de vida y hábitos de consumo, evidenciando las complejas estructuras que emergen en una sociedad capitalista neoliberal de una ciudad latinoamericana de segundo orden, y que definen grupos de demanda de hábitat específicos.
Se trabajó con la información relevada y procesada por el Instituto Nacional de Estadísticas y Censos (Indec), complementado con la información procesada por la Dirección de Estadística y Censos provincial y la Dirección de Estadísticas y Censos municipal, a partir de las cuales se reconstruyó cuantitativamente la evolución sociodemográfica en el territorio. Además, se incorporó información sobre estilos de vida y pautas de consumo elaborada y procesada por distintas entidades públicas 0 privadas. Esta construcción cuali y cuantitativa se complementó con un análisis sociológico de los cambios y permanencias detectados, basados en el análisis de bibliografía específica.

\section{Estructura urbana territorial: macrocefalia en una constelación de ciudades}

Con más de 40 millones de habitantes distribuidos en $2.780 .400 \mathrm{~km}^{2}$, Argentina tiene baja densidad de ocupación, superando apenas los 14 hab/km². Pero esta población no se distribuye de forma pareja en el territorio nacional. El $89.5 \%$ de su población es urbana, es decir, habita en aglomeraciones de más de 2.500 habitantes, ${ }^{1}$ tal como muestra el Gráfico 1. Ese gráfico también muestra que el $34 \%$ de la población urbana (casi 14 millones) habita en la megalópolis del área metropolitana de Buenos Aires, y el $9 \%$ en otros tres conglomerados de más de 1 millón de habitantes - Gran Córdoba, Gran Rosario y Gran Mendoza - configurando la estructura urbana-poblacional macrocefálica característica del país. 
Córdoba es el segundo centro urbano más poblado del país, después de Buenos Aires, con 1.329.604 habitantes; es también cabecera de la segunda región metropolitana, el Área Metropolitana de Córdoba (AMCBA), que reúne a más de 1.981 .737 habitantes en una superficie de más de 20.906 km².
La estructura macrocefálica se repite en Córdoba a escala provincial y metropolitana: La ciudad alberga más del $40 \%$ de la población provincial y el $67 \%$ de la población del AMCBA, como muestra el Gráfico 2.

El Gráfico 2 muestra cómo Córdoba repite los patrones de dispersión de la

\section{Gráfico 1 - Distribución de la población argentina en centros urbanos} (año 2010)

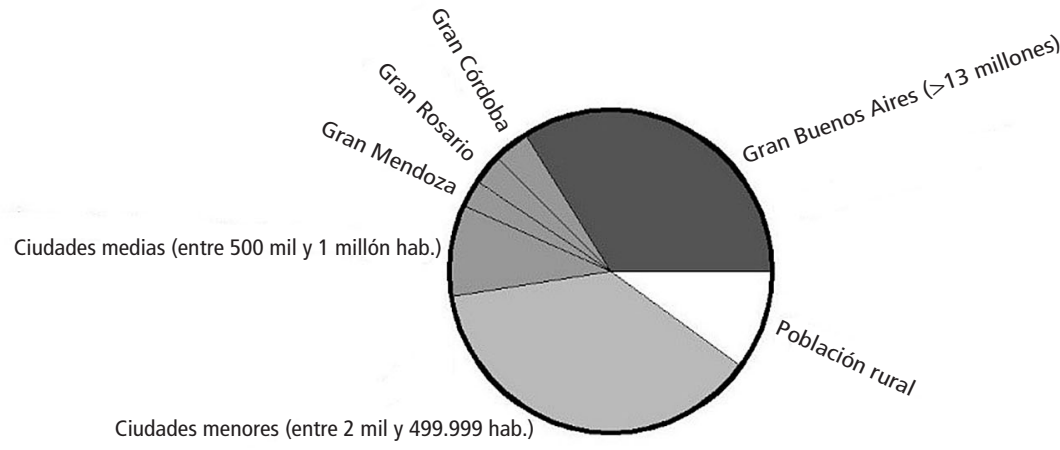

Fuente: Elaboración propia con base en datos de Indec (2012).

Gráfico 2 - Distribución de la población de la provincia de Córdoba (año 2010)

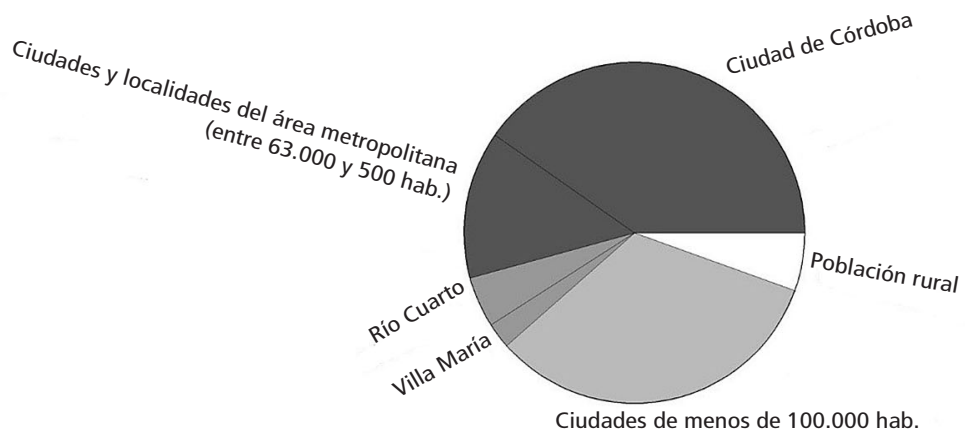

Fuente: Elaboración propia con base en datos de INDEC (2012). 
estructura urbana nacional. Los centros urbanos del AMCBA, distanciados en algunos casos hasta 49 km de Córdoba, configuran una constelación que se extiende por el territorio en un área aproximada de $20.906 \mathrm{~km}^{2} 2$ (Iplam, 2015) (ver Mapas 1 y 2).

Estos enormes centros urbanos difícilmente pueden ser explicados con base en el principio de jerarquía, la lógica que preside la dimensión y la localización relativa de los distintos centros de la red urbana, ${ }^{3}$ lo que evidencia otra contradicción. La cantidad de población y extensión territorial de estos centros urbanos son comparables a los grandes aglomerados que funcionan como centros de desarrollo y organización de procesos productivos a escala global. Sin embargo, su rol efectivo - dentro de una organización productiva nacional concentrada en la agroproducción exportadora - los semeja más a meros centros de servicios para el hinterland rural, verdadero motor económico del país. Tal como explica Mike Davis,

El modelo clásico del campo poseedor de una gran mano de obra y de la ciudad como fuente del capital se invierte en muchos lugares del Tercer Mundo, donde encontramos ciudades desindustrializadas poseedoras de una gran mano de obra, y regiones rurales con gran afluencia del capital. (2007, p. 28)

Como ejemplo, Córdoba genera el tercer Producto General Bruto (PGB) de Argentina después de Buenos Aires y de Rosario. Este distribuye entre el sector primario $(0,3 \%)$, secundario $(28,4 \%)$ - en el que destaca la importancia de las industrias autopartistas - y terciario (71,3\%); este último está orientado a servicios más o menos complejos a nivel provincial y regional, sobre todo de administración pública y actividades educativas. En el año 2010, el PGB fue de US\$14.387.609.000 lo que representa alrededor del 3,4\% del Producto Bruto Interno nacional (Dirección de Planeamiento, 1978; Secretaría de Planeamiento y Desarrollo Estratégico, 2014; Subsecretaria de Desarrollo Económico y Estratégico, 2012). Si se considera que la ciudad tiene el mismo porcentaje de la población total del país (3.31\%), es evidente que las economías de aglomeración, escala y urbanización no están desarrolladas de acuerdo a su rol como centro urbano de segundo nivel a escala nacional y regional.

Por otro lado, las actividades productivas y de servicios son originadas externamente. Las actividades industriales existentes son "importadas" de los centros de desarrollo globales, ${ }^{4}$ y funcionan como apoyo de la producción agroexportadora, u orientan su producción al mercado interno y regional. A pesar de albergar numerosas universidades públicas y privadas, Córdoba casi no posee actividades de innovación y desarrollo. Sus servicios son poco diversificados en relación con su jerarquía dentro del sistema urbano, o su escala demográfica-productiva (Walton, 1984, pp. 10-23; Jacobs, 1971; Geisse, 1986).

\section{Contraurbanización}

La concentración de la población en las grandes ciudades argentinas estuvo acompañada por las mayores tasas de crecimiento hasta casi fines del siglo XX; pero los datos muestran que en las dos últimas 
décadas este proceso se está revirtiendo: A partir de 1980, la tasa de crecimiento de las mayores ciudades de Argentina empieza a declinar (Torrado, 2003; Geller, 1978), al tiempo que las ciudades medias de las áreas metropolitanas ven aumentar sus tasas de crecimiento, en un fenómeno denominado contraurbanización, o urbanización revertida (Peralta, 2007), tal como muestran los Mapas 1 y 2.

Mapa 1 - Centros urbanos del AMCBA de acuerdo a cantidad de población (año 2010)

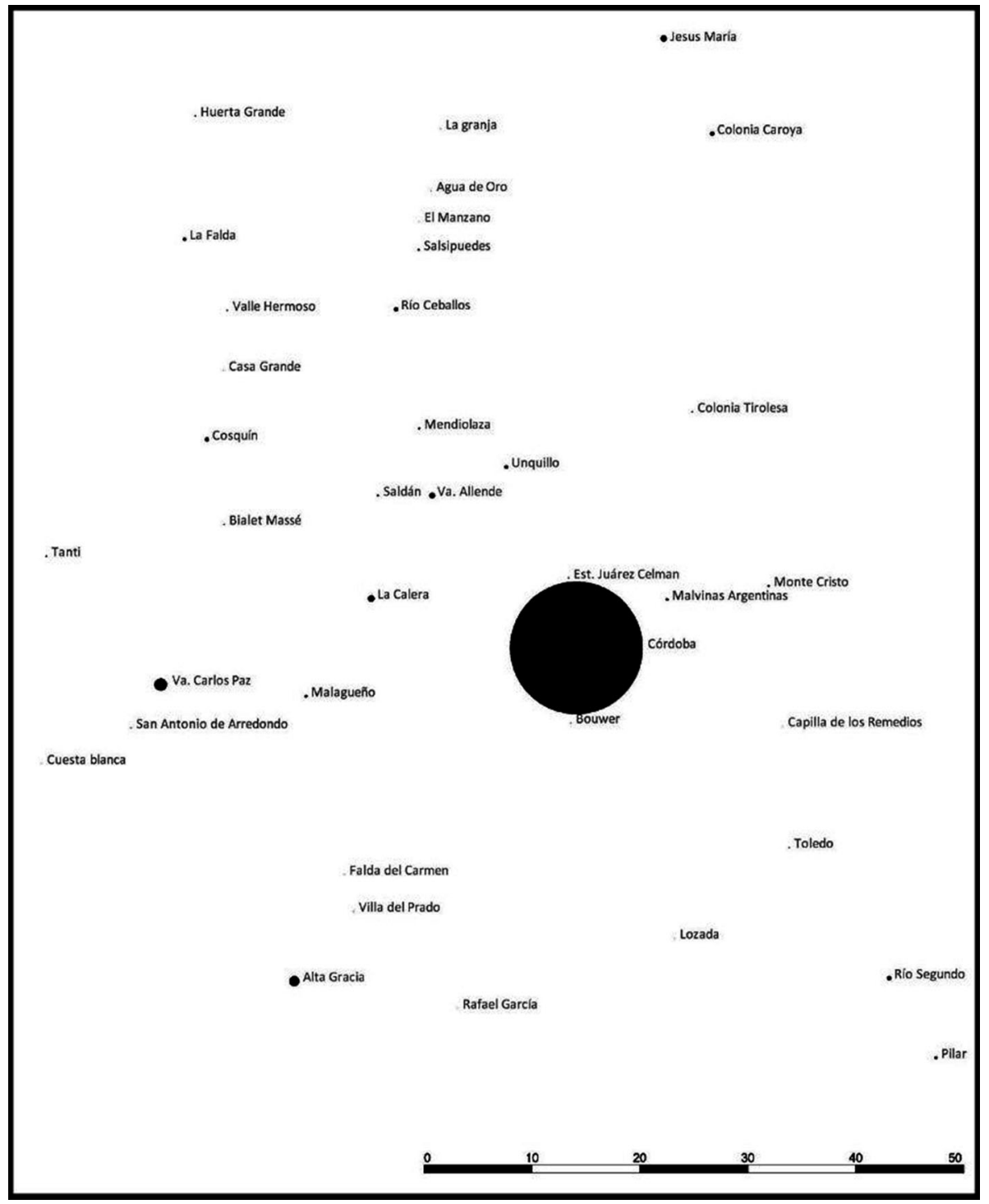

Fuente: Elaboración propia con base en datos de www.googlemaps.com.ar y la Dirección Provincial de Estadística y Censos de la Provincia de Córdoba (www.cba.gov.ar). 
Mapa 2 - Centros urbanos del AMCBA de acuerdo a sus tasas de crecimiento demográfico (año 2010)

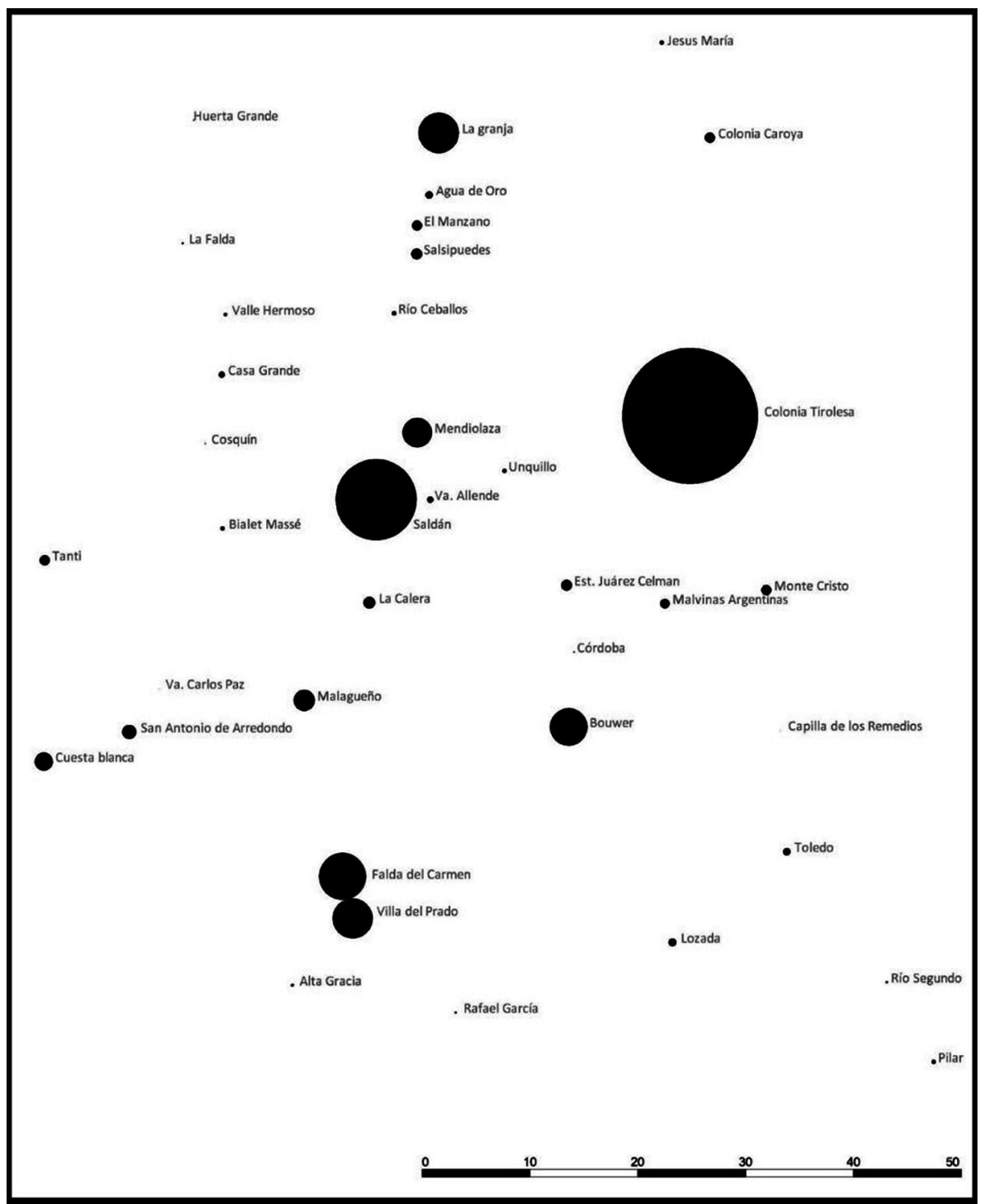

Fuente: Elaboración propia con base en datos de www.googlemaps.com.ar y la Dirección Provincial de Estadística y Censos de la Provincia de Córdoba (www.cba.gov.ar) 
Las causas de este proceso deben buscarse en las migraciones interurbanas, ya que las tasas de natalidad y mortalidad no muestran diferencias significativas entre localidades grandes y medias. El crecimiento natural no explica las diferencias registradas en la evolución de las respectivas poblaciones (Tecco, 2007).

En el caso de Córdoba, las migraciones hacia las localidades medianas del AMCBA no tienen como origen una crisis económica en la ciudad, sino los cambios de modelo en los procesos de poblamiento de las grandes áreas urbanas. Entre ellos, el más difundido es el paradigma de "vuelta al mundo rural" por parte de algunos segmentos de las poblaciones urbanas como una vuelta del "rechazo de la gran ciudad", a su densidad, artificialidad, mezcla social e inseguridad y poca calidad ambiental. Por otro lado, las inversiones en la red vial y de transporte, la penetración de las nuevas tecnologías de información y comunicación en amplios sectores de la población, y el teletrabajo facilitan la localización de la vivienda en zonas cada vez más alejadas de los lugares de trabajo (Peralta, 2007). También hay causas más profundas, relacionadas directamente con la dinámica del mercado de vivienda y suelo urbano de Córdoba en comparación con las localidades del AMCBA, en donde el valor de mercado del suelo urbano expulsa a sectores cada vez más amplios de la población a áreas periféricas (Boccolini, 2017).

Pero aunque Córdoba expulsa por año a casi 8.000 habitantes (Peralta, 2007), la dependencia del centro mayor por parte del resto no se supera, sino que se tensa cada vez más. Al ser la principal plaza laboral y de servicios del AMCBA, amplios sectores del área metropolitana funcionan sólo como "asentamientos dormitorio", y generan cada vez más traslados pendulares entre estas localidades y Córdoba (Tecco, 2007).

A pesar del proceso de contraurbanización, la población de la ciudad tiene una tasa de crecimiento positiva, del $0,38 \%$ anual. Mientras que otros centros urbanos como Buenos Aires incluso llegaron a tener tasas de crecimiento negativas entre 1991 y 2001 (Indec, 2012), las proyecciones realizadas hasta el año 2050 indican que cada año se sumarán a Córdoba aproximadamente 8.000 nuevos habitantes, debido al crecimiento vegetativo y a la inmigración desde el resto de la provincia, el país y otros países latinoamericanos principalmente.

\section{Transición demográfica avanzada como fenómeno demográfico}

La Argentina integra junto a Chile, Cuba y Uruguay, el grupo de países de Latinoamérica en una transición demográfica avanzada. Una causa importante en este proceso es el descenso de la fecundidad, $y$, en menor medida, el aumento de la expectativa de vida y el efecto de reestructuración (aunque cada vez menor) que produce el ingreso de población inmigrante (Pantelides y Moreno, 2009, p. 102), lo que se traduce en un crecimiento natural bajo, del orden del $1 \%$. Los datos de los Gráficos 3 y 4 confirman la tendencia al aumento del peso relativo de la población adulta mayor ${ }^{5}$ $y$, a la vez, dan cuenta de menores niveles de natalidad (Indec, 2012). 


\section{Gráfico 3 - Evolución de la relación entre grupos etarios en Córdoba}

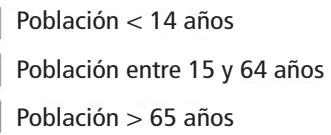

Población > 65 años

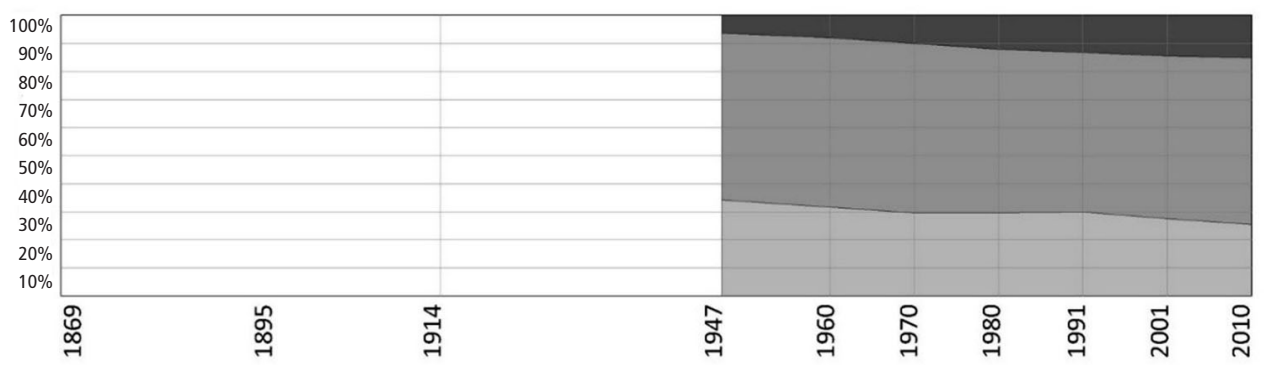

Fuente: Elaboración propia con base en datos de Subsecretaria de Desarrollo Económico y Estratégico (2012).

Gráfico 4 - Estructura demográfica del país, por sexo, edad y origen (año 2010)
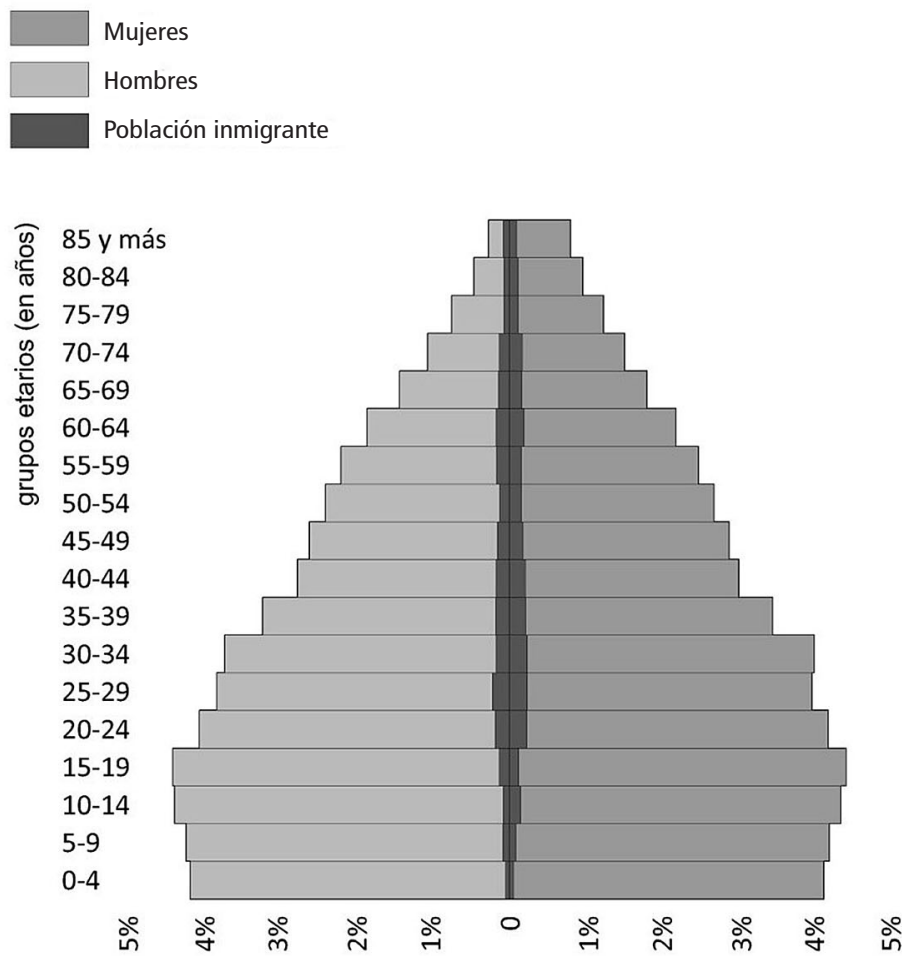

Fuente: Indec (2012). 
La estructura etaria de la población de Córdoba refleja en parte porque las ciudad tiene una de las tasas de fecundidad más bajas del país, mientras que la provincia de Córdoba es una de las cuatro con mayor porcentaje de población mayor a 65 años (11,2\% en 2010), una tendencia que se profundizará en el futuro. Según los datos censales, la población aumentó 14 veces entre 1869 y 1991, mientras que la población mayor de 60 años aumentó 74 veces en el mismo período (Peralta, 2006).

\section{Migraciones urbanas}

Como ya se ha mencionado, la población argentina ha recibido importantes aportes de las sucesivas migraciones ocurridas durante los últimos 140 años, que se miden no sólo en términos meramente cuantitativos, sino también cualitativos, en cuanto a aportes culturales, técnicos, tecnológicos, etc. y se refleja en los valores culturales de la población argentina.

Con respecto a Córdoba, se estima que un $90 \%$ de la población desciende de los inmigrantes europeos de principios del siglo XX, principalmente italianos y españoles, aunque la ciudad cuenta también con considerables minorías judías, árabes, armenias, japonesas, chinas y coreanas, que forman colectividades con fuerte presencia institucional.

Por otro lado, desde mediados del siglo XX la inmigración proveniente de países sudamericanos, principalmente Paraguay, Perú y Bolivia, ha cobrado mayor importancia; en los últimos 15 años Córdoba se ha consolidado como uno de los centros que más inmigrantes ha atraído con respecto al resto del país (Indec, 2012 , p. 107). Hoy el 2,3\% de la población de la ciudad es extranjera, principalmente de estos países latinoamericanos.

A esto se suma el hecho de que la ciudad es sede de cinco instituciones universitarias, con más de 180.000 personas entre alumnos, docentes y empleados (lo que representa el $14 \%$ de la población de la ciudad). ${ }^{6}$ Por ello recibe un constante flujo de estudiantes provenientes del Noreste y Noroeste argentino, la Patagonia, ciudades del interior provincial y de países sudamericanos como Bolivia, Perú y Paraguay. Esto representa una gran cantidad de población inmigrante de determinado rango etario (18 a 35 años), de carácter temporal y renovación permanente. La incidencia de la población estudiantil puede observarse en la pirámide poblacional del Gráfico 5, perturbando la estructura de transición demográfica avanzada que se correspondería con la del resto del país que muestra el Gráfico 4.

El impacto de la población estudiantil inmigrante no se limita a aumentar la cantidad de personas que asisten a la universidad. Luego de egresar, gran parte de esos inmigrantes elijen instalarse en la ciudad durante los primeros años de su carrera profesional o indeterminadamente.

La inmigración de países limítrofes también contribuye (aunque en menor medida), ya que este grupo también tiene una marcada presencia de personas en edad productiva (Ver Gráfico 5, a la izquierda). Los inmigrantes y sus descendientes se dedican a actividades que van desde los estudios universitarios y posgrado ${ }^{7}$ 


\section{Gráfico 5}

(Izquierda) Estructura demográfica de la ciudad por sexo y edad (Derecha) Estructura demográfica de la ciudad por edad y origen (año 2010)
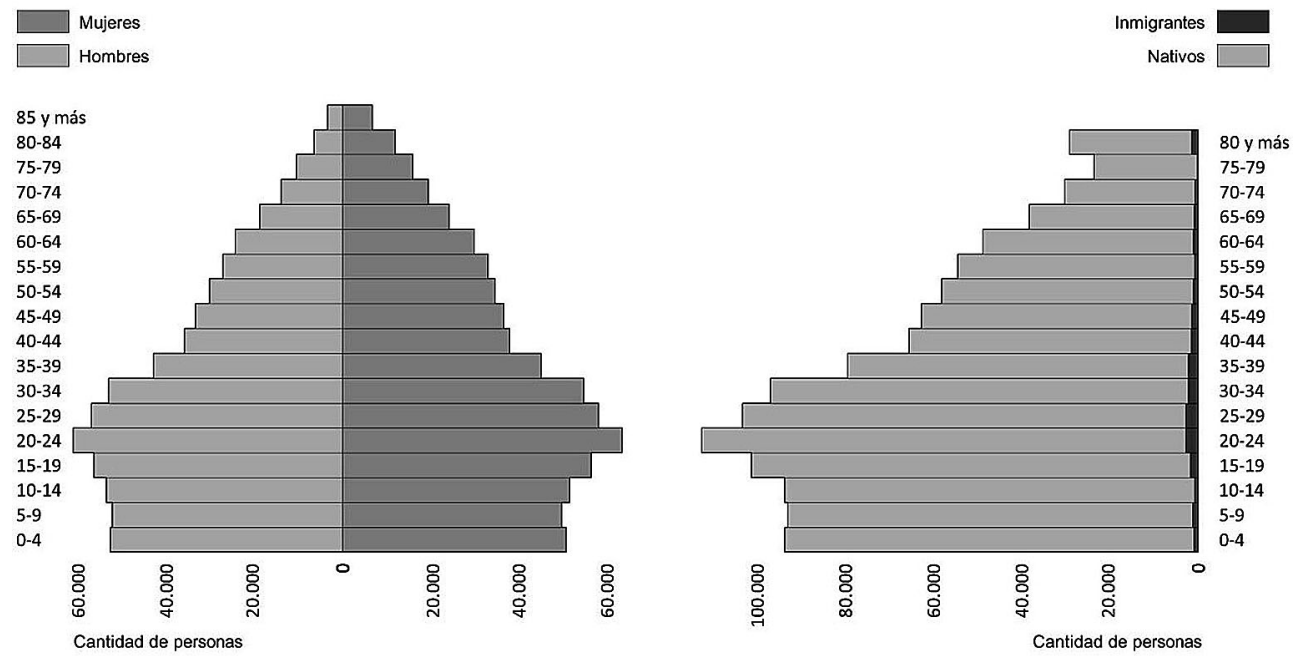

Fuente: Secretaría de Planeamiento y Desarrollo Estratégico (2014).

hasta la construcción, la gastronomía y la horticultura, insertándose en el mercado laboral de forma más o menos formal.

Estas particularidades son factores de peso al evaluar la estructura demográfica de la ciudad, y las demandas específicas de hábitat. El período durante el cual lo individuos incrementan su demanda por servicios habitacionales es entre los 25 y 60 años, ya que la demanda en los primeros años de vida es prácticamente nula, y muy reducida en la tercera edad (Peralta, 2006). 


\section{Transición demográfica avanzada en su dimensión socio-cultural}

La transición demográfica avanzada que está transitando la población de la ciudad tiene también variables de carácter cualitativo desde un punto de vista sociológico, que reflejan la situación a escala nacional. Siguiendo a Susana Torrado (2003), se está produciendo una mayor individualización de los ciudadanos, concebida como un apartamiento progresivo de los mandatos y sujeciones institucionales (de la religión, las doctrinas políticas, las organizaciones sindicales, etc.) que favorece la expansión de la esfera privada sobre el ámbito público.

También hay cambios más profundos, en la estructura de la familia "post-moderna". Se rechaza el cumplimiento de los roles tradicionales de esposo/esposa y madre/padre impuestos por la tradición, y el rol asignado a hombres y mujeres en lo que respecta a la participación laboral y al sustento de la familia. Los hombres están menos compelidos a constituir el principal proveedor de recursos; las mujeres ven disminuir sensiblemente su dependencia económica como efecto de su mayor escolarización y de su mejor inserción laboral. Se valorizan formas de realización personal distintas a "tener hijos"; la institución matrimonial pierde su rol de protección, seguridad y "umbral iniciático de la sexualidad femenina".

Estos cambios [...] remiten a una demanda, explícita o implícita, de autonomía personal, de valoración del ámbito privado, de desvalorización de los lazos de dependencia respecto a las instituciones y a las personas. [...] Los actores poseen un mayor control de su destino individual y familiar en razón de nuevos valores que aprueban esa autonomía e inducen cambios trascendentales en el derecho de familia, en el sistema tributario, en las políticas sociales. (Torrado, 2003)

A continuación, veremos cómo estos cambios se reflejan en la estructura demográfica de los hogares argentinos en general y cordobeses en particular.

\section{Los hogares argentinos}

En los últimos años, los hogares ${ }^{8}$ en Argentina han comenzado un proceso de cambios en su estructura, tamaño y perdurabilidad en el tiempo (ver Tabla 1). Las transformaciones sociodemográficas, económicas y culturales tienen correlaciones directas con nuevas formas de habitar que aparecen de forma simultánea. Las causas son varias.

En primer lugar, Pantelides y Moreno (2009, pp. 133-136) mencionan cambios en los patrones de nupcialidad y conyugalidad. Éstos son provocados por el

[...] retraso del calendario y cambio en las modalidades de entrada a las uniones conyugales, con aumento de la importancia de las uniones consensuales en detrimento del peso de las uniones legales; aumento de las separaciones y divorcios y de las segundas o sucesivas uniones y/o matrimonios que, a su vez, pueden dar lugar a las familias ensambladas. 
En segundo lugar, mencionan la disminución en el tamaño de las familias, debido al continuado descenso de los niveles de fecundidad (o al mantenimiento de un bajo nivel de fecundidad alcanzado con anterioridad). Esto se debe, entre otras causas a las políticas de educación sexual y la difusión de métodos anticonceptivos (Pantelides y Moreno, 2009, p. 144), pero más directamente frente a cambios culturales de planificación familiar y de cuestiones que hacen al desarrollo personal de la población femenina. La paridez media final ha descendido desde 3,1 hijos por mujer en el 2001 a 2,9 hijos en el 2010 (Indec, 2012, p. 197). Además, la disminución del tamaño de los hogares se debe también al proceso de nuclearización de los hogares familiares, evidenciado en el aumento de hogares unipersonales y monoparentales.

El preconcepto de que los hogares unipersonales correspondían a personas mayores pierde validez frente a las nuevas situaciones detectadas. La vida en solitario es un estado que puede ser transitado en diferentes estadios de la vida. Los hogares de personas solas de jóvenes son en su mayoría varones solteros; en los hogares de los adultos solos predominan los hombres divorciados, ya que las mujeres divorciadas suelen convivir con sus hijos (Torrado, 2003); y en los hogares de adultos mayores, las viudas (Aguirre, 2004, pp. 20-22).

Córdoba repite estas tendencias (ver Tabla 2). La cantidad de personas por hogar ha descendido de 3,45 en el año 2001 a 3,16 en el año 2010. La consecuencia más evidente de acuerdo a los datos cuantitativos es que los hogares biparentales con hijos - la tipología históricamente más frecuente - van perdiendo peso relativo con respecto a los hogares unipersonales, los monoparentales con hijos y las familias extendidas; y el promedio de personas por hogar se reduce.

Tabla 1 - Cantidad de hogares por tipo de estructura familiar, para el total del país (Censos 2001 y 2010)

\begin{tabular}{|c|c|c|c|c|c|c|}
\hline & & & \multirow{2}{*}{\multicolumn{2}{|c|}{2001}} & & \\
\hline & & & & & \multicolumn{2}{|c|}{2010} \\
\hline & & & unid. & $\%$ & unid. & $\%$ \\
\hline \multicolumn{3}{|l|}{ Total hogares } & 10.073 .625 & 100,00 & 12.171 .675 & 100,00 \\
\hline \multicolumn{3}{|l|}{ Unipersonales } & 1.511 .123 & 15,00 & 2.156 .771 & 17,72 \\
\hline \multirow{4}{*}{ Multipersonales } & \multirow{3}{*}{ Familiares } & Nucleares & 6.366 .314 & 63,20 & 7.542 .209 & 61,97 \\
\hline & & Extendido (con familiares) & 1.970 .509 & 19,56 & 2.199.296 & 18,07 \\
\hline & & Compuesto (con no familiares) & 142.985 & 1,42 & 159.365 & 1,31 \\
\hline & \multicolumn{2}{|c|}{ No familiares } & 82.694 & 0,82 & 114.034 & 0,94 \\
\hline
\end{tabular}

Fuente: Elaboración propia con base en datos de INndec (2012). 
Tabla 2 - Cantidad de hogares por tipo de estructura familiar, para Córdoba (Censos 1991, 2001 y 2010)

\begin{tabular}{|c|c|c|c|c|c|c|c|c|}
\hline & & & & & & & & \\
\hline & & & \multicolumn{2}{|c|}{1991 * * } & \multicolumn{2}{|c|}{2001} & \multicolumn{2}{|c|}{2010} \\
\hline & & & unid. & $\%$ & unid. & $\%$ & unid. & $\%$ \\
\hline \multicolumn{3}{|l|}{ Total hogares } & 318.093 & 100,00 & 359.404 & 100,00 & $\mathrm{~s} / \mathrm{d}$ & $s / d$ \\
\hline \multicolumn{3}{|l|}{ Unipersonales } & 39.569 & 12,44 & 52.805 & 14,69 & $s / d$ & $s / d$ \\
\hline \multirow{4}{*}{ Multipersonales } & \multirow{3}{*}{ Familiares } & Nucleares & 245.700 & 77,24 & 214.545 & 59,69 & $s / d$ & $\mathrm{~s} / \mathrm{d}$ \\
\hline & & Extendido * & 35.800 & 11,25 & 74.832 & 20,82 & $s / d$ & $s / d$ \\
\hline & & Compuesto (con no familiares) & 54.756 & 17,21 & 4.788 & 1,33 & $s / d$ & $s / d$ \\
\hline & \multicolumn{2}{|c|}{ No familiares } & 19.556 & 6,15 & 8.488 & 2,36 & $\mathrm{~s} / \mathrm{d}$ & $\mathrm{s} / \mathrm{d}$ \\
\hline
\end{tabular}

Fuente: Elaboración propia con base en datos de Indec (2012).

Pero para profundizar en este proceso de transformación de la estructura de los hogares, es necesario hacer una aclaración con respecto a los datos aportados por el Indec, y su metodología de recolección, que podrían interferir - paradójicamente - en la visibilización de parte de la realidad de los hogares argentinos.

Durante la recolección de datos en los censos, cada modelo familiar se determina con base en la relación de los integrantes con una persona de referencia, el "jefe de hogar", que explícita o implícitamente suele responder al modelo patriarcal y recae por lo tanto con mayor frecuencia en el miembro varón adulto de mayor edad - generalmente padre y/o esposo - (Pantelides y Moreno, 2009, pp. 138-139; Aguirre, 2004, p. 26). Esto conlleva la invisibilización de otras realidades, más o menos novedosas, tales como los núcleos secundarios en el interior del hogar, las familias ensambladas, ${ }^{9}$ la diferencia entre hijos/as biológicos/as y adoptivos/as, o las uniones homosexuales ${ }^{10}$ (y su posible encubrimiento bajo la declaración y registro de otro vínculo - de parentesco o no - socialmente más aceptado o no estigmatizado).

También invisibiliza el hecho de que en la mayoría de los hogares ya no hay una sola persona que dirige la vida doméstica y familiar, sino que la organización suele responder a los dos miembros de la pareja (en estructuras biparentales) o varias personas, familiares o no, pertenecientes a la misma o a distintas generaciones. La obsoleta autoridad del "jefe de hogar" se ha diluido, debido a transformaciones socio-culturales pero también económicas. Gracias a situaciones económicas del contexto global y nacional, aumenta la cantidad de aportantes al hogar, ya sea la esposa/pareja de hecho, los hijos en edad de trabajar, o incluso la integración de varios aportes económicos en un hogar multipersonal no familiar. 
Otra situación que debe tenerse en cuenta en cuanto a los datos recogidos en los censos es la relación entre géneros en la estructura del hogar. La mayor participación de la mujer en el sostén económico del hogar es posible debido a nuevas condiciones culturales de mayor igualdad entre géneros, pero también a la necesidad ineludible de sumar ingresos económicos al grupo familiar, ya sea por disminución del poder adquisitivo de los salarios individuales o como "seguro" frente a la inestabilidad laboral de los miembros económicamente activos del grupo familiar. Por lo tanto, la incorporación de más mujeres al mercado de trabajo "no implica necesariamente una mejora en el ejercicio de sus derechos y en sus condiciones de vida o la de sus familias" (Pantelides y Moreno, 2009, p. 154; véase también Topalov, 1979, p. 47 y Ss., y Davis, 2007, pp. 203-209).

Por otro lado, y con respecto a los cambios significativos en los patrones de nupcialidad y conyugalidad, hay una tendencia creciente a retrasar la edad de contraer matrimonio y tener el primer hijo (Indec, 2012, pp. 196-211), o directamente no ser éstas opciones en la planificación familiar, sobre todo en mujeres jóvenes de estratos socioeconómicos medios y alto y con mayor nivel educativo (Aguirre, 2014, pp. 9-10). Las uniones consensuadas son más comunes, ${ }^{11}$ al igual que la procreación dentro de esas uniones o fuera de ellas (familias monoparentales), o la crianza de hijos con parejas sin vínculo paternal biológico (familias ensambladas).

Las familias monoparentales de jefatura femenina se revelan, además, como las estructuras familiares más vulnerables:
[...] constituyen un universo particularmente vulnerable en razón de su propia composición. La misma impone restricciones a la capacidad de generar ingresos - ya que la madre suele ser la única perceptora -, al tiempo que debe realizar las labores domésticas que demanda el grupo familiar. Esta circunstancia se agudiza en ciclos económicos depresivos y es una traba para la acumulación en momentos de auge, ya que estas mujeres tienen poco margen de maniobra para compatibilizar conductas que les permitan mantener o aumentar sus ingresos - por ejemplo, aumentando las horas de trabajo - sin producir el consiguiente efecto negativo sobre su familia. (Torrado, 2003)

Este hecho significaría una diferencia crucial con los hogares monoparentales masculinos, pero también con los hogares unifamiliares de hombres divorciados que reciben esporádicamente la visita de sus hijos, y, por qué no, los hogares ensamblados con hijos producto de uniones anteriores de la mujer, pero que también esporádicamente suman los hijos del hombre producto de una unión anterior. Esto no es un fenómeno nuevo - particularmente entre los estratos sociales más bajos y en ciertas provincias donde históricamente su nivel es alto. La novedad es su incremento en regiones y entre sectores sociales donde la convivencia fuera del matrimonio no estaba difundida, es decir, las grandes ciudades y las provincias del área central y pampeana (Pantelides y Moreno, 2009, p. 154), entre las que se encuentra Córdoba.

Otra singularidad que emerge en la ciudad son los hogares multi-personales no 
familiares, con un crecimiento continuo en relación a hogares familiares. En Córdoba, estos representan un porcentaje tres veces mayor al del resto del país (ver Tabla 2). Son hogares formados por personas conocidas entre sí, amigos, colegas, que conviven en estructuras más o menos permanentes en distintas etapas de la vida. Pueden estar relacionados con la reducción de gastos fijos en la vivienda, principalmente atribuídos a la población estudiantil universitaria, pero que últimamente son vinculado a nuevas estructuras familiares "superadoras" de la pareja con o sin hijos, sin distinguir grupos etarios o laborales.

La diversificación de las estructuras familiares es acompañada también de otro proceso complejo que no ha sido relevado en los censos. El aumento de las estructuras familiares sucesivas, incluso simultáneas, que una persona forma a lo largo de su vida. Los estados "tradicionales" por las que una persona pasaba a lo largo de su vida eran el primer núcleo familiar - biparental - como hijo/a; el segundo núcleo como cónyuge y madre/padre; el tercero, el "nido vacío" en la "tercera edad" seguido de un breve período de viudez donde se vivía en soledad o con algún hijo y su familia. En la actualidad, cada uno de estos estados se desdobla en varios, se diluye con otros o desaparece, de acuerdo a las circunstancias de cada individuo. Otras pueden presentarse varias veces a lo largo de la vida de una persona, casi como "bucles" (Ascher, 2004, pp. 36-39).

Esto refleja no sólo transformaciones culturales y sociales, sino estrategias de supervivencia atribuidos tradicionalmente a los sectores más débiles que son puestas en práctica por grupos de población cada vez más amplios. Algunas de estas prácticas, como la elección de vivir solo o sola, son fenómenos más propios de sectores sociales relativamente acomodados, ${ }^{12}$ en tanto que las familias extendidas, en las que conviven personas emparentadas en distinto grado, son mayoritariamente un reflejo de las estrategias asociativas y solidarias para disminuir los costos de vida de otros sectores sociales.

La importancia de incluir estas situaciones familiares en esta investigación va más allá del mero relevamiento enumerativo. Poner en relieve los nuevos patrones de vida es un intento de superar la invisibilización que hasta ahora han sufrido, en un proceso que tiene correlación directa con el de "expulsión" desarrollado por Sassen (2015).

El término expulsiones se aplica a las categorías de situaciones para las cuales las variables tradicionales han perdido toda pertinencia, entre ellas las que son producto de la adaptación a nuevas condiciones laborales, económicas, políticas y culturales. Pueden englobarse aquí tanto las organizaciones familiares no tradicionales como la volatilidad familiar, las pautas de vida y estructuras interpersonales, los nuevos roles en los grupos multi-personales y las nuevas expectativas de vida "familiar".

Se expulsa a estas categorías invisibilizándolas en el registro oficial de los censos y en el campo de conocimiento; se les quita validación, reconocimiento, derechos, necesidades. Pero la falta más grave es que, mientras los grandes actores del mercado inmobiliario privado en Córdoba ya ha desarrollado herramientas identificar estos nuevos grupos de consumidores y seleccionar los más adecuados a su esquema de producción 
(Observatorio de tendencias de hábitat, 2009), las políticas públicas de hábitat y desarrollo territorial siguen invisibilizando a los grupos más vulnerables (Tecco, 2007).

Son entonces los sin poder, sin visibilidad, los sectores cuyas demandas de hábitat no pueden ser resueltas eficientemente por el mercado, que busca la máxima rentabilidad, cuyas nuevas estructuras de vida emergentes deben ser puestas en relieve para definir integralmente las políticas públicas territoriales y urbanas, ya que además de las variables económicas y sociales (inversiones, pensiones, distribución del ingreso, capacidad de ahorro, eficiencia del población activa, etc.), los cambios demográficos y en las estructuras familiares son variables determinantes en la demanda habitacional (Peralta, 2006). Otras variables determinantes del hábitat urbano son las pautas productivas, laborales y de consumo estudiadas a continuación.

\section{Polarización socioeconómica a inicios del siglo XXI}

Los modelos de desarrollo económico implementados en el país durante sus primeros 170 años fueron en primer lugar el agroexportador y posteriormente la industrialización por sustitución de importaciones (Dalle, 2010; Torrado, 2003). Ambos modelos contribuyeron de diversas formas a formar una estructura social integrada, con gran permeabilidad entre grupos.

Hacia 1960-1970, Argentina aún se distinguía en el contexto latinoamericano por las oportunidades de ascenso social que había brindado a las personas de origen de clase popular y por la amplitud de las clases medias y una clase trabajadora fabril con una posición económica consolidada, sustentada en niveles salariales altos y acceso a derechos sociales comparativamente altos (Dalle, 2010). Córdoba es un ejemplo de esto, al consolidarse como un centro industrial de gran importancia al comenzar la segunda mitad del siglo XX. ${ }^{13}$

Sin embargo, a partir de 1970 se configura el proceso neoliberal de apertura y desregulación de la economía que, entre otras cosas, produjo la desaparición de gran parte de las pequeñas y medianas empresas, ${ }^{14}$ y disminuyó el empleo industrial tanto en puestos técnicos, administrativos como obreros calificados (Sautu, 1997, cit. en Dalle, 2010). Con esto aumentó la precarización laboral, el trabajo por cuenta propia, como "contratista" y no como parte de la planta permanente de una empresa, y la desocupación tanto en los estratos de clase media como en la clase obrera. Por otro lado, los sectores más altos de la estructura social se vieron favorecidos por la creación de empleo de gestión altamente calificado de compañías privadas de bienes y servicios que se instalaban en el país (Dalle, 2010; Svampa, 2001; Aguirre, 2004).

Como resultado de este proceso, "se ha dado paso a una creciente segmentación al interior de la clase media y a un aumento importante de los estratos más bajos", "en la cual las oportunidades de acceso a los recursos de la modernidad y a los mercados laborales más competitivos se torna crecientemente diferente" (Mora y Araujo, 2002, pp. 5 y 33). Mientras que los sectores más bajos aumentan -consecuencia de la movilidad descendente de las clases medias bajas-, la polarización de la 
clase media se produce debido a varias causas: Por un lado, nuevos empleos de alta calificación permitieron el ascenso inter-generacional desde sectores medios (cuadros técnicos y administrativos y pequeños comerciantes), formando "un segmento de clase media-alta ligado a corporaciones financieras y empresas de servicios multinacionales con altos salarios y un estilo de vida suntuoso".

Por otro lado, sectores asalariados que pertenecen a sindicatos con fuerte poder de negociación se han beneficiado con aumentos muy superiores a los obtenidos por aquellos perfiles históricamente emparentados con la clase media típica. Estos sectores, llamados "la nueva clase media trabajadora", ${ }^{15}$ están formados por portuarios, metalúrgicos, aceiteros, telefónicos o petroleros.

Al mismo tiempo, parte de la clase media asalariada (empleados y cuadros técnicos de la administración y empresas estatales privatizadas) tuvo que adoptar ocupaciones de servicios no calificados y gran precariedad laboral (Aguirre, 2004, p. 5), con cada vez menos oportunidades de movilidad social ascendente, y donde la mayor exposición directa a las condiciones del mercado - sin mediación sindical -, provocan una tendencia descendente tanto de las remuneraciones como de las oportunidades económicas, que pueden empujarlos rápidamente a posiciones socioeconómicas más bajas (Mora y Araujo, 2002, p. 36). Gran parte de la clase media autónoma (profesionales independientes, pequeños y medianos comerciantes, industriales y productores rurales) tuvo que cerrar sus empresas en el marco del proceso de concentración y centralización de capital que implicó la apertura y desregulación de la economía, separando más y más al estrato medio alto del medio bajo. Por último,

[...] la clase media-alta conformado por profesionales, gerentes y propietarios de capital aumentó el nivel de herencia inter-generacional reteniendo para sí sus privilegios de clase. Muchos hijos de propietarios de capital pasaron a ser profesionales, gerentes y cuadros directivos intermedios principalmente en el sector privado. (Dalle, 2010)

Estas transformaciones son reflejadas por la población de Córdoba, en cuanto a aumento de la precariedad laboral y polarización de la clase media. Sin embargo, los datos recogidos por el Indec en la última década muestran una tendencia reparadora de estos procesos, en la que la pobreza estructural disminuyó en casi todo el territorio nacional, (Indec, 2012, pp. 309-317) y los sectores medios, aun conservando la segmentación producida en décadas anteriores, comenzaron a crecer, reduciendo la importancia relativa de los sectores bajos y marginales, tal como muestra el Gráfico 6.

A pesar de esto, hay procesos de transformación en el mercado laboral (De Mattos, 2010; Ascher, 2004; Muxí, 2010, 2013; Echeverría, 1995) que, lejos de desaparecer, amplían sus efectos sobre las pautas de la población. Estos procesos son parte de los procesos de reorganización económico-productivos a escala global cuya influencia es cada vez más evidente a escala local (De Mattos, 2010; Sassen, 1999, 2010), sobre todo por el alto porcentaje de profesionales universitarios y terciarios que 
Gráfico 6 - Evolución de la estratificación socioeconómica de la población del país

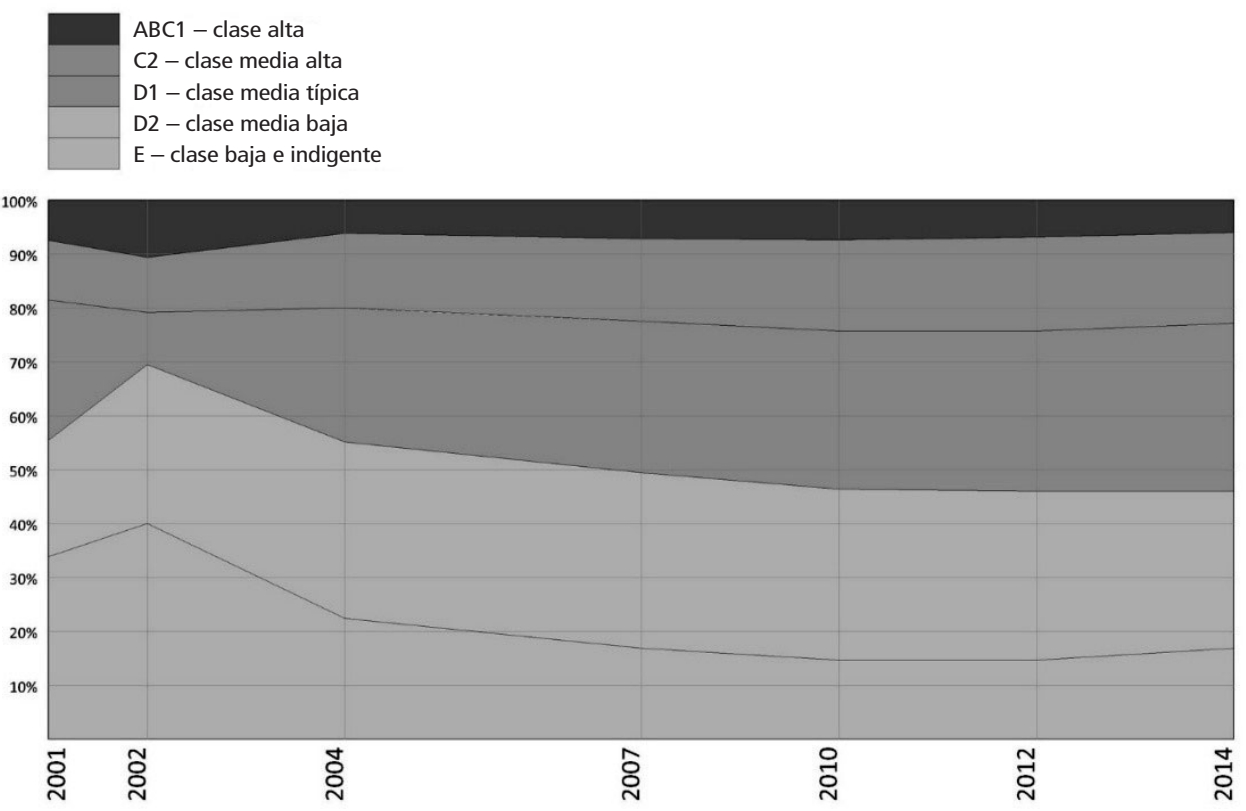

Fuente: Elaboración propia con base en datos de Mora y Araujo (2002: 41) y la Asociación Argentina de Marketing (www.aam-ar.org.ar).

se forman en los establecimientos educativos de la ciudad y luego buscan insertarse en el mercado laboral local.

Es el caso, por ejemplo, del sub-empleo y la precariedad laboral. Ya no es la norma la "carrera" laboral dentro de una misma empresa, ni siquiera en el mismo rubro. Se incorporan al mercado cada vez más jóvenes profesionales especializados como trabajadores "externos", cuenta propia o free-lance. Aumenta la tendencia a cambiar de empleo cada dos años aproximadamente, y los "ascensos" no sólo se dan dentro de la empresa, sino que suelen ser mejoras laborales conseguidas al cambiar de empleo. Esto puede entenderse como parte del proceso de crecimiento relativo del empleo en sectores terciarios y la "heterogeneidad y polarización de los regímenes de empleo contemporáneos, derivados principalmente de las características del sector terciario, el cual está sometido a procesos muy diferentes de aquellos que caracterizan a la industria manufacturera" (Mingione, 1998, p. 6, cit. en De Mattos, 2010, p. 52).

Esta situación flexibiliza la situación laboral para ambas partes. En primer lugar, libera al empleador de muchos costos como el aguinaldo, las vacaciones pagas y los 
aportes previsionales y de salud - que son en realidad beneficios sociales conquistados para los trabajadores. Como contraparte, los "empleados externos" tienen un horario flexible, pueden combinar varios empleos a la vez y, en algunos casos, pueden incluirse formas de teletrabajo. ${ }^{16}$ El lugar de trabajo ya no está exclusivamente en la empresa, sino que puede estar en la vivienda, en un estudio compartido, o en otra zona geográfica. El auge del teletrabajo "transfronterizo" es una prueba de ello. Aparecen los trabajadores "golondrina" que distribuyen su residencia en dos o más lugares (del país o del mundo) alternándolas más o menos periódicamente de acuerdo a sus actividades laborales.

Es evidente que la lógica de reproducción de la división social y económica que se está desarrollando genera la retroalimentación que transforma los patrones socio-culturales y laborales de la población. Las tendencias de movilidad y transitoriedad de las estructuras familiares tienen su reflejo en la movilidad laboral (Ascher, 2004, pp. 38-43). Los cambios en los patrones laborales promueven los cambios en los patrones familiares y los vínculos sociales y viceversa. Emergen nuevos patrones de organización estructurados por las nuevas condiciones del contexto. Los nuevos medios de transporte y de almacenamiento de personas, informaciones y bienes liberan a individuos, empresas e instituciones de límites espaciales y temporales. La co-presencia o la proximidad no son ya necesarias para muchos intercambios y prácticas sociales.

Esta movilidad laboral, sin embargo, está programada para realizarse en lo posible dentro del estrato socio-productivo original. Las barreras entre estratos socio-económicos son cada vez más impenetrables, y se tiende a la concentración del "capital social" al interior de cada uno de estos grupos.

\section{Discusión de resultados: impacto de estas transformaciones en la demanda de hábitat en Córdoba}

La reconstrucción de las características sociodemográficas de la población desarrollada en este trabajo muestra varias tendencias que estructuran cambios en la demanda de hábitat urbano.

En primer lugar, es innegable que la tendencia a consolidar una red urbana macrocefálica, tanto a escala nacional como regional (AMCBA) es inducida por las macroestructuras productivas y políticas. La aglomeración de servicios y actividades de base en los centros mayores es causa y consecuencia de la fuerte gravitación de población hacia ellos, y de una red de infraestructura y transporte muy concentrada, en relación a un territorio tan extenso. La concentración en los centros urbanos parece no generar economías de escala y de aglomeración acordes, lo que se evidencia al comparar la productividad de los centros urbanos entre sí y con el área rural.

Sin embargo, la aglomeración de personas, y el proceso de crecimiento acelerado que la originó, si han generado una ciudad congestionada, con un área central híper densa, y frecuentemente colapsada, en relación a las áreas periféricas, poco consolidadas y con déficits generalizados en equipamiento y servicios (Boccolini, 2017; Iplam ciudad, 2015). 
Al mismo tiempo, y a pesar de que los procesos que determinan la condición macrocefálica no han disminuido su impacto, emerge en las últimas décadas la tendencia centrífuga de contraurbanización, que desafía al modelo concentrado. Todavía, la estructura urbana resultante no genera una constelación urbana de nuevos centros en el territorio, o el desarrollo jerárquico de los centros medios dentro de cada área metropolitana. La contraurbanización en el AMCBA dilata las estructuras urbanas existentes sobre el territorio rural y replica en los centros urbanos metropolitanos el proceso de congestión de la estructura urbana que tuvo lugar en Córdoba hace más de medio siglo.

Esto plantea varios interrogantes. En principio, ¿es posible que la contraurbanización sea una oportunidad para equilibrar el sistema macrocefálico territorial? Si se reconoce el potencial del fenómeno para descentralizar las regiones metropolitanas, y lograr un desarrollo más equilibrado en el territorio, es necesario plantear políticas específicas que aprovechen el potencial de la contraurbanización, minimizando sus posibles inconvenientes. Porque, ¿cuál es su impacto en los pequeños y medianos centros urbanos del AMCBA? La estructura urbana de estas aglomeraciones puede que no soporte este crecimiento poblacional acelerado, replicando el proceso de crecimiento acelerado próximo al colapso de Córdoba. Además, ¿qué implica este modelo de urbanización para el desarrollo y gestión de la infraestructura de trasporte, comunicación, servicios y gobernanza a nivel urbano y regional, en cuanto a lógicas de eficiencia y sostenibilidad?
Por otro lado, ¿cuál es su impacto en Córdoba? Parece no producir un estancamiento productivo, ya que a pesar de la contraurbanización, sigue teniendo un peso considerable en la estructura económica regional y nacional. Pero es cierto que la población abandona áreas ya urbanizadas, y se relocaliza en áreas periféricas donde será necesario construir extensiones de la infraestructura de servicios básicos (Peralta, 2007). Entonces, ¿qué impacto tendrá el aumento de inmuebles no utilizados en la ciudad construida? ¿Prevalecerá la pauperización de grandes áreas urbanas, o serán renovadas, gentrificadas, 0 revitalizadas? Más aún, ¿cuál es el impacto de extender la urbanización cada vez más, perdiendo territorio periurbano de reserva natural o agrícola-productivo?

Sumado a ello, deben cobrar relevancia los procesos de polarización al interior de cada clase socioeconómica, que reducen las posibilidades de relación o movilidad entre distintos grupos. La segregación socioeconómica se traduce eventualmente en distintas pautas de vida (producción, educación, consumo) y el tejido social se segmenta cada vez más. Es necesario definir cómo impacta esto en la territorialización del hábitat urbano, donde la segregación se traduce en ciudades cada vez más fragmentadas (Peralta, 2006).

Además, como se ha visto, las transformaciones socio cultural que implican cambios en las estructuras domésticas, determinan nuevas categorías cuali y cuantitativas de hogares urbanos. Los grupos familiares tradicionales se disgregan y reorganizan en grupos más atomizados, de 
estructura más flexible, menos jerárquica y menos estable en el tiempo y en el territorio. La categoría "familiar" ya no sea la base a partir de la cual se explican todas las estructuras, y el ámbito residencial ahora incluye prácticas no domésticas. El aumento de hogares no familiares, la sucesión de cada vez más estructuras familiares en la vida de una persona, la movilidad territorial dada por el trabajo o situaciones personales, son variables que incidirán necesariamente en la definición tipológica del hábitat urbano (mayor flexibilidad, tipologías no jerarquizadas, nuevos programas espaciales y funcionales). La movilidad personal y la volatilidad de las estructuras familiares también inciden en el régimen de acceso y tenencia de la vivienda, desafiando el modelo actual de una vivienda familiar propia para toda la vida adulta. ${ }^{17}$

El gran porcentaje de población entre 18 a 35 años en la ciudad, que representa no sólo población económicamente activa, sino también el grupo con mayor demanda de hábitat, refleja además con mayor intensidad estas transformaciones en cuanto a estructuras de hogar y pautas de vida. Pero también es necesario considerar los hogares más vulnerables que emergen en estas tendencias. Por un lado, la población de adultos mayores y ancianos (mayores de 55 años), de gran importancia en la ciudad (que seguirá creciendo en importancia en los próximos años); por otro lado, las familias monoparentales de madres solteras; finalmente, los hogares que emergen en la población inmigrante que llega a la ciudad, con o sin asistencia de parientes, allegados 0 el Estado. Estos grupos sociales desarrollan necesidades específicas de reformulación tipológica, accesibilidad doméstica y a los servicios urbanos, y, nuevamente, de instrumentos de acceso y tenencia, que no sólo influyen en la producción de hábitat a futuro, sino que generan la necesidad de refacción y adecuación de viviendas existentes.

Se plantea entonces el interrogante sobre las condiciones que debieran tener las propuestas de hábitat urbano para dar respuesta a los requerimientos espaciales, funcionales y jurídicos específicos que generan estas transformaciones en los hogares, en el ámbito doméstico y como parte indivisible de la ciudad, y en qué medida las políticas públicas de producción de hábitat, y el mercado inmobiliario privado, los responden.

Este trabajo cumple entonces su objetivo de reconstruir una base sobre la cual pueden concretarse otras instancias de investigación, en cuanto estudio de políticas de hábitat, mercado inmobiliario y patrones de territorialización que resultan de estas tendencias. Pero como aporte específico de este trabajo, se reconoce que visibilizar estos cambios socioeconómicos resulta fundamental para entender los procesos de territorialización de la ciudad; y tal como lo evidencia la discusión de los resultados obtenidos, también es imprescindible para analizar críticamente las políticas públicas de hábitat urbano actuales, y orientar el desarrollo de los futuros instrumentos de ordenamiento, no sólo de la producción de hábitat urbano sino de la urbanización a escala metropolitana y regional. 


\section{Sara María Boccolini}

Universidad Nacional de Córdoba, Facultad de Arquitectura, Urbanismo y Diseño, Instituto de Investigación de la Vivienda y del Hábitat. Córdoba, Argentina.

saraboccolini@gmail.com

\section{Notas}

(1) Esta es la población mínima para que una aglomeración sea considerada como "centro urbano" de acuerdo al Indec.

(2) Esta superficie empequeñece la de conglomerados urbanos globales como el área metropolitana de París, de sólo 14.518 km².

(3) Si se desea profundizar en las implicaciones de la estructura macrocefálica, se recomienda revisar los trabajos de Friedmann, 1966; Gilbert, 1978; Browning y Roberts, 1978; a escala nacional, ver Ministerio de Planificación Federal, Inversión Pública y Servicios (2011); a escala provincial y metropolitana ver Dirección de Planeamiento Urbano (1981a, 1981b), e Iplam ciudad (2015).

(4) Como parte del proceso de reorganización de la producción y consumo a escala global.

(5) $14.3 \%$ en 2010 , con una estimación de $24.8 \%$ en 2050 . Esto se acentúa debido a una disminución simultánea en el porcentaje de población menor a 14 años (25.5\% en 2010 , de un $28.3 \%$ en 2001)

(6) Entre las instituciones universitarias está la Universidad Nacional de Córdoba, segunda más antigua de Latinoamérica y la tercera en cantidad de alumnos entre las universidades públicas nacionales.

(7) Más asequibles, en cuanto a costos directos e indirectos, en Argentina en comparación con otros países de Latinoamérica.

(8) La definición de "hogar" que brinda el Censo 2010 es la de grupo de personas que viven bajo un mismo techo y comparten los gastos de alimentación (Indec, 2012, p. 171).

(9) "Ya que los/las hijos/as de uniones anteriores de uno o ambos cónyuges del núcleo principal o bien son registrados/as como 'hijo/a' del jefe/a o bien se los registra como 'otros parientes', en cuyo último caso el hogar resultará clasificado como extendido" (Pantelides y Moreno, 2009).

(10) Esta situación empieza a visibilizarse a partir del Censo 2010, el que registra que el $0.33 \%$ de las parejas relevadas son formadas por personas del mismo sexo, pero aún no pueden considerarse como datos fidedignos cuantitativos.

(11) Mientras que el 55,3\% de las personas mayores de 14 años declaran vivir en pareja, sólo el 35,8\% está casado civilmente. Esto significa una reducción con respecto al año 2001, donde el $42.6 \%$ de las personas mayores de 14 años se declaraban casadas. Hay una tendencia decreciente de matrimonios: del total de personas mayores de 14 años en pareja, en el año 1991 estaban casadas el $82 \%$; en el 2001 , el $73 \%$ y en el 2010 , sólo el $61.2 \%$. 
(12) Los hogares unipersonales son más frecuentes en los dos quintiles superiores de ingreso, ya que implican la capacidad de afrontar los gastos del hogar individualmente. La situación se complejiza si se incluyen diferentes pautas de vida definidas por la orientación sexual o particularidades dadas por la población temporal (estudiantes o trabajadores tipo "golondrina" o simplemente turistas extranjeros que no son contabilizados en el censo).

(13) Ya se ha mencionado la importancia que tienen aún hoy, las industrias autopartistas en el PGB de la ciudad.

(14) Habiendo crecido bajo la protección del mercado interno, no pudieron soportar la competencia de las importaciones.

(15) "La cultura del sindicalismo activo, orientada a defender posiciones económicas relativamente favorables, se ve amenazada por la escasa competitividad de los sectores donde ella perdura, donde los niveles de capacitación y las aptitudes laborales tienden a colocar a sus miembros en desventaja competitiva" (Mora y Araujo, 2002, p. 36).

(16) La modalidad de teletrabajo no tiene una penetración homogénea en el mercado laboral. Debido a sus características, es más frecuente en los sectores de servicios empresariales como actividades inmobiliarias, software y servicios informáticos y seguros (Datos del Observatorio Tripartito de Teletrabajo, www.trabajo.gov.ar). El desarrollo de la net-economy y las nuevas tecnologías de información y comunicación (TIC) influye en la creación de cada vez más empleos de este tipo (Ascher, 2004, pp. 48-50).

(17) En el año 2010, más del $60 \%$ de los hogares son propietarios de la vivienda y la parcela donde esta está construida (Indec, 2012). Las políticas de vivienda en Córdoba y Argentina serán estudiadas en instancias futuras de esta investigación.

\section{Referências}

AGUIRRE, R. (2004). Familias urbanas del Cono Sur: transformaciones recientes. Argentina, Chile y Uruguay, Cepal.

ASCHER, F. (2004). Los nuevos principios del urbanismo: el fin de las ciudades no está a la orden del día. Madrid, Alianza.

BOCCOLINI, S. (2017). Regulação urbana em Córdoba. ¿’para uma cidade sustentável e inclusiva? Urbe (en prensa).

BROWNING, H. y ROBERTS, B. (1978). Urbanización, transformación sectorial y utilización de la mano de obra en Latinoamérica: Una interpretación histórica desde la perspectiva internacional. En: Ensayos histórico-sociales sobre la urbanización en América latina. Buenos Aires, Sociedad interamericana de planificación.

DALLE, P. (2010, ene). Estratificación social y movilidad en Argentina (1870-2010). Revista de Trabajo, v. 6, n. 8 .

DAVIS, M. (2007). Planeta de ciudades miseria. Madrid, Akal. 
DE MATTOS, C. (2010). Modernización capitalista y transformación metropolitana en América Latina: cinco tendencias constitutivas. En: América Latina: cidade, campo e turismo. São Paulo, Consejo Latinoamericano de Ciencias Sociales.

DIRECCIÓN DE PLANEAMIENTO URBANO (1978). Esquema de ordenamiento urbano. Córdoba, Municipalidad de Córdoba

(1981a). Esquema Director de Ordenamiento Metropolitano-EDOM 1981-2000. Córdoba, Municipalidad de Córdoba.

(1981b). PlanDeMet: Plan de Desarrollo Metropolitano de Córdoba. Documento síntesis. Córdoba, Municipalidad de Córdoba.

ECHEVERRÍA, J. (1995). Cosmopolitas Domésticos. Buenos Aires, Editorial Anagrama S.A.

FRIEDMANN, J. (1966). Regional development policy: a case study of Venezuela. M. I. T. Press.

GEISSE, G. (1986). Tres momentos históricos de la ciudad latinoamericana del siglo XX. Eure, v. XIII, n. 38.

GELLER, L. (1978). Estrategias de crecimiento y la distribución espacial de la población. México DF, Cepal.

GILBERT, A. (1978). La planificación ante la primacía y las grandes ciudades de América Latina. Una crítica de la bibliografía. En: Ensayos histórico-sociales sobre la urbanización en América latina. Buenos Aires, Sociedad interamericana de planificación.

INDEC (2012). Censo Nacional de Población, Hogares y Viviendas 2010 Censo del Bicentenario Resultados definitivos, Serie B, n. 2. Buenos Aires, Indec.

IPLAM CIUDAD (Ed.). (2015). Pensar y construir la ciudad. Córdoba, Departamento de publicaciones/ FAUD-UNC.

JACOBS, J. (1971). La economía de las ciudades. Barcelona, Península.

MINISTERIO DE PLANIFICACIÓN FEDERAL, INVERSIÓN PÚBLICA Y SERVICIOS (2011). Plan Estratégico Territorial Avance II: Argentina Urbana. Buenos Aires, Letra Viva.

MORA Y ARAUJO, M. (2002). La estructura social de la Argentina: evidencias y conjeturas acerca de la estratificación actual. Santiago de Chile, Naciones Unidas.

MUXÍ, Z. (2010). Revisar y repensar el habitar contemporáneo. RiURB - Revista Iberoamericana de Urbanismo, n. 3, pp. 4-9.

OBSERVATORIO DE TENDENCIAS DE HÁBITAT (2009). Nuevas formas de habitar. Valencia, Conselleria de Industria Comercio e Innovación.

PANTELIDES, E. y MORENO, M. (eds.). (2009). Situación de la población en la Argentina. Buenos Aires, Programa Naciones Unidas para el Desarrollo/PNUD/UNFPA.

PERALTA, C. (2006). La ciudad periurbana. Discusiones acerca de la extensión urbana periférica y el espacio público. En: Marengo, C. (ed.). La periferia de Córdoba: cuestiones sobre hábitat urbano. Córdoba, Dirección de publicaciones de la FAUD-UNC.

(2007). Migraciones internas y destinos urbanos. Evolución del saldo migratorio de la Ciudad de Córdoba. 1914-2001. IX JORNADAS DE ESTUDIOS DE POBLACIÓN. Aepa, IX. 
SASSEN, S. (1999). La Ciudad Global: Nueva York, Londres, Tokio. Eudeba. (2010). Territorio, autoridad y derechos. Madrid, Katz Editores. (2015). Expulsiones. Brutalidad y complejidad en la economía global. Madrid, Katz Editores.

SECRETARÍA DE PLANEAMIENTO Y DESARROLLO ESTRATÉGICO (2014). Córdoba. Una ciudad en cifras. Guía estadística de la ciudad de Córdoba 2014. Córdoba, Municipalidad de Córdoba.

SUBSECRETARIA DE DESARROLLO ECONÓMICO Y ESTRATÉGICO (2012). Córdoba. Una ciudad en cifras. Guía estadística de la ciudad de Córdoba julio 2012. Córdoba, Municipalidad de Córdoba.

SVAMPA, M. (2001). Los que ganaron: la vida en los countries y barrios privados. Buenos Aires, Biblos.

TECCO, C. (2007). Gestión metropolitana y equidad social. En: Construyendo el Estado Nación para el crecimiento y la equidad. Buenos Aires.

TOPALOV, C. (1979). La urbanización capitalista: algunos elementos para su análisis. Mexico DF, Edicol.

TORRADO, S. (2003). Historia de la familia en la Argentina moderna (1870-2000). Buenos Aires, Ediciones de la Flor.

WALTON, J. (ed.). (1984). Ciudades y sistemas urbanos: economía informal y desorden espacial. Buenos Aires, Consejo Latinoamericano de Ciencias Sociales.

Texto recebido em 13/set/2016 Texto aprovado em 28/out/2016 
